Evidence summaries

\title{
Human epidermal growth factor receptor 2 borderline mortality in breast cancer patients: Evidence from surveillance, epidemiology, and end results program population-based study
}

\author{
Atanu Bhattacharjee ${ }^{\mathrm{a}, *}$, Tapesh Bhattacharyya ${ }^{\mathrm{b}}$, Abin Thomas ${ }^{\mathrm{c}}$ \\ ${ }^{a}$ Centre for Cancer Epidemiology, The Advanced Centre for Treatment, Research and Education in Cancer (ACTREC), Tata Memorial Centre, Navi Mumbai \\ 410210, India \\ ${ }^{\mathrm{b}}$ Department of Radiation Oncology, Tata Medical Centre, Kolkata, India \\ c Indian Institute of Technology, Dhanbad, India
}

\section{A R T I C L E I N F O}

\section{Article history:}

Received 19 October 2017

Accepted 17 December 2017

Available online 3 January 2018

\begin{abstract}
A B S T R A C T
Background: Breast cancers with Human Epidermal Growth Factor Receptor 2 (HER2) borderline outcomes are very puzzling for patient management. To whether HER2 borderlines are having different prognostic status in comparison to HER2 positive/negative.

Methods: This is a study among women diagnosed with invasive breast cancer from 1973 to 2014 who were identified in the Surveillance, Epidemiology, and End Results (SEER) of 9 registries database and having diagnosed with HER2 status $(\mathrm{N}=102167)$, i.e. HER2 positive $(\mathrm{N}=14678)$, HER2 negative $(\mathrm{N}=85354)$ and HER2 borderline $(\mathrm{N}=2135)$.

Results: This Surveillance, Epidemiology, and End Results (SEER) study represents the largest report of prospective invasive breast cancer with HER2 status till five years from the date of registration. The five years survival rate among HER2 borderline was found completely separate with HER2 positive and HER2 negative patients. In several subtypes of analysis, it has been found that status of survival among HER borderline patients is significantly poor than HER2 positive and HER2 negative. The likelihood of death in HER2 borderline is found high (15.06\%) in comparison to HER2 positive (14.24\%) and HER2 negative (6.49\%) at the end of five years. Different types of metastasis like Bone, Brain, Liver, and Lung are also observed with the higher rate in HER2 borderline in comparison to HER2 negative.
\end{abstract}

(C) 2017 Published by Elsevier, a division of RELX India, Pvt. Ltd on behalf of INDIACLEN.
Conclusion It can be concluded that HER2 borderline breast cancer patients are required to take specific treatment management, not in line with HER2 positive or HER2 negative.

\section{Introduction}

There is an existing variation on treatment success in breast cancer with different factors with Age, Race etc. ${ }^{1,2}$ Recently, organizations like are traced about the development of new research model to adopt in the clinical practice for the development of value-added cancer therapy. ${ }^{3,4}$ However, there is good success towards breast cancer particularly in the developed countries but breast cancer remains the leading cause among all types of female cancer deaths. ${ }^{5}$ The management of invasive breast cancer is decided through the evaluation of Human Epidermal

\footnotetext{
* Corresponding author.

E-mail address: abhattacharjee@actrec.gov.in (A. Bhattacharjee).
}

Growth Factor Receptor 2 (HER-2) status. There is a discrepancy on HER-2 status detection between manual Immuno Histo Chemistry (IHC) and Fluorescence In Situ Hybridization(FISH). The IHC is performed through a special staining process on frozen or fresh breast cancer tissue. It is performed to test the presence of HER2 receptors in cancer cell. The FISH test is performed on breast tissue to count the extra copies of HER2 gene. The outputs provide through FISH is considered as 'gold standard'. ${ }^{6}$ The gene amplification is considered to classify the status of the FISH as positive or negative. However, FISH and IHC both provides the HER2 status with borderline status as well. Once it becomes difficult to classify the gene amplification as positive/negative then it is classified as "borderline". It is very important to test the hypothesis that whether the borderline HER-2 patients are clinically equivalent with presence or absences of HER-2 status or not. The Surveillance, Epidemiology, and End Results (SEER) Program was started in 1973 by the National Cancer Institute. It is a populationbased cancer surveillance program. Nearly $30 \%$ of US populations are covered under this program and more than $98 \%$ incidence of 
cancer cases were covered. ${ }^{7}$ Information on demographics profile, site of the tumor, type of treatment and survival status of the patients is recorded. Patients treated with breast cancer and their HER-2 status was recorded in the SEER database.

The objective of this study is to check the performance of HER-2 borderlines in comparison to HER-2 positive/negative. In this study, we analyze the 'borderline' group of tumors identified by FISH. The median overall survivals (OS) of patients with HER-2 positive, negative are compared with HER-2 borderlines.

\section{Methods}

This study analyzed data on HER2 status in breast cancer patients from the SEER registries. The dataset are accessible from https://seer.cancer.gov/data/citation.html through signed Research Data Agreement Form. Data is based on the populationbased cancer registries that participate in the SEER program under National Cancer Institute (NCI). ${ }^{5}$ The SEER program covers the data from states of Connecticut, Iowa, New Mexico, Utah, Hawaii, Louisiana, Kentucky, New Jersey, and California and from Alaska Natives in Alaska, in addition to the metropolitan areas of Detroit, Michigan; San Francisco-Oakland, San Jose-Monterey, and Los Angeles County, California,Atlanta, Georgia, and Seattle-Puget Sound, Washington. Around $30 \%$ of U.S. populations are covered under SEER program. Different types of patient's demographic information, diagnosis confirmation, and the first course of treatment, the extent of disease, morphology, and active patient follow-up for vital status including the cause of death are included in this program. The cause of death is derived from death certificate of a resident when cancer is listed as a cause of death. The reported incidence and death status data are available nearly two years after the end of the calendar year. Till now the incidence data till December 2014 is available. The HER2 status of all breast cancer patients is included in this study. The influence of HER2 status on survival outcomes is considered. The survival analysis is performed through the Cox Proportional Hazard ( $\mathrm{CPH}$ ) and Accelerated Failure Time (AFT) models.

\section{Results}

Total of 102167 patients in the participating SEER registries of invasive breast are included in this study (Table 1 ). The distribution pattern of age, race and tumor size of these patients are like: Age (<40 years, i.e. 3127 [9.95\%]), Age(40-49 years i.e. 5401 [17.19\%]),
Age(50-59 years i.e. 8256 [26.28\%]), Age(60-69 years i.e. 7603 [24.2\%]), Age( $70-79$ years i.e. 4436 [14.12\%]) and Age ( $>80$ years i.e. 2585 [8.23\%]). The tumor sizes are classified as $<5 \mathrm{~mm}, 5-10 \mathrm{~mm}$, $10-15 \mathrm{~mm}, 15-20 \mathrm{~mm}, 20-40 \mathrm{~mm}$ and $>40 \mathrm{~mm}$. All histological grades are presented with grades 1,2 and 3 respectively. The further demographic classification based on Grade1, Grade2 and Grade3 of the breast cancer are provided in Tables 4-6 (Supplementary File S1) respectively. The Progesterone Receptor (PR) and Estrogen Receptor (ER) status of the patients in all grades are given in Table 1 and Tables 4-6. Comparison of the occurrence of HER-2 positive, HER-2 negative and HER-2 borderline in different Age groups, tumor sizes and Race are given with pvalue. It shows that there is not any difference about occurrence of HER-2 positive, HER-2 negative and HER-2 borderline in any subtypes of Age, Race and Tumor size except race for Grade 1 . The analysis provides the comparison of the bone, liver. Lung and brain metastasis in HER-2 positive, HER-2 negative and HER-2 borderline in Table 2. It provides that there is a significant occurrence of HER-2 status in different metastasis. It shows that occurrence of different metastasis is relatively higher in HER-2 borderline group in comparison to HER-2 positive, HER-2 negatives. Occurrence of different metastasis in ER and PR status also presented graphically. It shows that different types of metastasis are relatively higher in ER borderline in comparison to ER positive and ER negative. Same

Table 2

Different Types of Metastasis on HER2 Status, ER Status and PR Status for the treated patients from $2010+$.

\begin{tabular}{lllll}
\hline Types of Metastasis & HER2+ & HER2- & HER2 B & P-value \\
\hline Bone & $634(4.25 \%)$ & $2226(2.55 \%)$ & $80(3.66 \%)$ & $<2.2 \mathrm{e}-16$ \\
Brain & $94(0.63 \%)$ & $204(0.23 \%)$ & $12(0.55 \%)$ & $<2.2 \mathrm{e}-16$ \\
Liver & $401(2.69 \%)$ & $708(0.81 \%)$ & $22(1.01 \%)$ & $<2.2 \mathrm{e}-16$ \\
Lung & $317(2.12 \%)$ & $961(1.10 \%)$ & $31(1.42 \%)$ & $<2.2 \mathrm{e}-16$ \\
& & & & \\
ER+ ER- ER B P-value & & & & \\
Bone & $2441(2.8 \%)$ & $481(2.85 \%)$ & $1(1.37 \%)$ & 0.7046 \\
Brain & $189(0.22 \%)$ & $118(0.7 \%)$ & $0(0.0 \%)$ & $<2.2 \mathrm{e}-16$ \\
Liver & $743(0.85 \%)$ & $378(2.24 \%)$ & $1(1.37 \%)$ & $<2.2 \mathrm{e}-16$ \\
Lung & $901(1.03 \%)$ & $401(2.38 \%)$ & $1(1.37 \%)$ & $<2.2 \mathrm{e}-16$ \\
& & & & \\
PR+ PR- PR B P-value & & & & \\
Bone & $1964(2.59 \%)$ & $945(3.35 \%)$ & $7(4.76 \%)$ & $2 \mathrm{e}-10$ \\
Brain & $140(0.18 \%)$ & $164(0.58 \%)$ & $2(1.36 \%)$ & $<2.2 \mathrm{e}-16$ \\
Liver & $547(0.72 \%)$ & $567(2.01 \%)$ & $5(3.40 \%)$ & $<2.2 \mathrm{e}-16$ \\
Lung & $713(0.94 \%)$ & $582(2.06 \%)$ & $3(2.04 \%)$ & $2.2 \mathrm{e}-16$ \\
\hline
\end{tabular}

Table 1

Patient Demographics by HER2, ER and PR status( $N=102167)$.

\begin{tabular}{|c|c|c|c|c|c|c|c|c|c|c|}
\hline \multirow[t]{2}{*}{ Category } & \multicolumn{10}{|c|}{ All Grades } \\
\hline & HER2+ & HER2- & HER2B & ER+ & ER- & ERB & $\mathrm{PR}+$ & PR- & PRB & P-value \\
\hline \multirow[t]{6}{*}{ Age } & 1532 & 4234 & 109 & 4312 & 1551 & 5 & 3752 & 2104 & 9 & 0.65 \\
\hline & 2615 & 12239 & 296 & 12404 & 2698 & 15 & 11391 & 3694 & 16 & 0.07 \\
\hline & 4215 & 20131 & 525 & 23012 & 4513 & 10 & 17356 & 7410 & 37 & 0.91 \\
\hline & 3527 & 23911 & 569 & 23841 & 4112 & 15 & 20576 & 7336 & 36 & 0.22 \\
\hline & 1839 & 16066 & 405 & 15967 & 2304 & 19 & 13796 & 4456 & 25 & 0.19 \\
\hline & 950 & 8773 & 231 & 8618 & 1313 & 5 & 7304 & 2604 & 16 & 0.35 \\
\hline \multirow[t]{4}{*}{ Race } & 1820 & 8777 & 255 & 7874 & 2956 & 7 & 6449 & 4376 & 7 & 0.02 \\
\hline & 1867 & 9087 & 192 & 9344 & 1787 & 6 & 8149 & 2968 & 13 & 0.81 \\
\hline & 42 & 230 & 4 & 241 & 35 & 0 & 213 & 62 & 1 & 0.07 \\
\hline & 10949 & 67260 & 1684 & 67995 & 11713 & 56 & 59364 & 20198 & 118 & 0.2 \\
\hline \multirow[t]{5}{*}{ Tumor Size } & 1237 & 7380 & 211 & 7693 & 1108 & 8 & 6539 & 2230 & 13 & 0.63 \\
\hline & 1687 & 16529 & 340 & 16752 & 1775 & 5 & 14917 & 3579 & 22 & 0.2 \\
\hline & 4280 & 29768 & 711 & 30050 & 4649 & 17 & 26524 & 8130 & 45 & 0.27 \\
\hline & 4574 & 21505 & 554 & 20984 & 5595 & 22 & 17977 & 8571 & 29 & 0.34 \\
\hline & 2900 & 10172 & 319 & 9945 & 3364 & 17 & 8218 & 5094 & 30 & 0 \\
\hline
\end{tabular}

*153 patients ER status and 249 patients ER and PR status were unknown in this cohort of 102167, A total of 4023 patients Grades were unknown. 
trend also observed in case of PR status as well. It also provides information that PR borderlines are present with higher chance of having metastasis in comparison to others.

The survival analyses are conducted through $\mathrm{CPH}$ and AFT models. However, it is observed that the proportional hazard assumptions are not followed in different time points. Hence as alternative of $\mathrm{CPH}$, the AFT models are explored. The AFT models are explored through exponential distribution and Weibull distribution.

Table 3 shows CPH model and AFT models outputs for different risk factors according to the hazard ratio (HR) and P-value between HER2, ER and PR status. Under a different combination of HER 2 positive, HER 2 negative, HER 2 borderline, ER positive, ER negative, ER borderline, PR positive, PR negative, PR borderline the performance of $\mathrm{CPH}$ is defined under Model 1. The performance of AFT through the assumption of an exponential distribution with the different combination is defined as Model 2. The Model 3 is defined as AFT with Weibull distribution. It provides different outputs with models through the combination of different subtypes of positive, negative and borderline of HER 2, ER and PR status.

Different types of metastasis and death occurrence for the follow-up period of the five years are given in Fig. $1 \mathrm{~A}$ and B. It shows that the occurrence of death at the end of five years is comparatively high for HER2 borderline patients in comparison to HER2 positive and HER 2 negative. The corresponding event rates are also provided in Table 3 . The survival duration comparison between HER 2 positive, HER 2 negative and HER 2 borderline are given in Fig. 2. It represents the higher rate of death in the HER2 borderline group of patients. The proportion of death at the end of five years in HER2 positive observed with $6.49 \%, 14.24 \%$ in HER 2 negative and $15.06 \%$ in HER 2 borderlines respectively. The comparison of death occurrence between different ER and PR status are documented in Tables 7 and 8 respectively. However, these types of trend were not observed in ER and PR groups (Fig. 3).

The likelihood of death in HER2 borderline is high (15.06\%) in comparison to HER2 positive (14.24\%) and HER 2 negative (6.49\%) at the end of five years, based on 1973-2014 data. The treatment steps for HER 2 borderline in not clear, but HER 2 borderline suffers more in comparison to other types of HER2 status. Different types of metastasis like Bone, Brain, Liver, and Lung are also observed with the higher rate in HER2 borderline in comparison to HER2 negative. However, it was lower than HER 2 positive. It is required to take specific treatment management for HER 2 borderline patients.

It is assumed that the hazard rates between HER2 positive, HER2 negative and HER2 borderline patients are same. The hazard rates between HER2 positives, HER2 negatives and HER2 Borderlines are performed through $\mathrm{CPH}$ hazard assumption. The HER status of the patients was considered as covariates in CPH model. The different grade-wise stratified analysis is also performed with the same assumption. As an alternative to $\mathrm{CPH}$, the AFT models were also performed. The 'aftgee' package in open source software $R$ ( $R$ Cran project $R$ version 3.4.2) is considered to perform this analysis. Table 3 reveals that HER 2 negatives were having better survival in comparison to HER 2 positive with Hazard Ratio 0.9 $(0.85,0.95)$ through Model 1 . Models defined as Model1 are performed under $\mathrm{CPH}$ assumption. The same inference also observed through Model 2 and Model 3 between comparison with HER2 negative and HER2 positives by AFT. The hazard ratio found higher in HER2 borderline in comparison to HER2 positive by Model 1 with hazard ratio 1.05(0.97,1.13). However, the comparison between HER2 borderline and HER2 negative shows that significantly higher hazard ratio in HER2 borderline in comparison to HER2 positive. Tables 7 and 8 (Supplementary File S1) provides in grade wise comparison of Hazard ratio on different status of ER and PR.

\section{Discussion}

The CPH considered that the duration of survival is a function of multiple covariates. It assumes that the impacts of covariates are constant over time. ${ }^{8}$ It is documented that nearly $5 \%$ of studies investigate the proportional hazard assumption. ${ }^{9}$ In this work, we faced that the duration of OS was not followed the proportional hazard assumption between the groups. As an alternative of proportional hazard, the AFT has also performed with an assumption of Exponential and Weibull distribution. Only Exponential and Weibull distribution assumptions are considered for AFT due to limited parametric function available in 'aftgee' package. In all of the choices, we did not get any major difference of statistical inference through different choices of distributional assumption either by CPH or AFT. The reason is the presence of the high volume of sample size in this analysis. We believed that our data in follow with central limit theorem due to their size with high volume. It is obvious that unreliable and bias result may occur

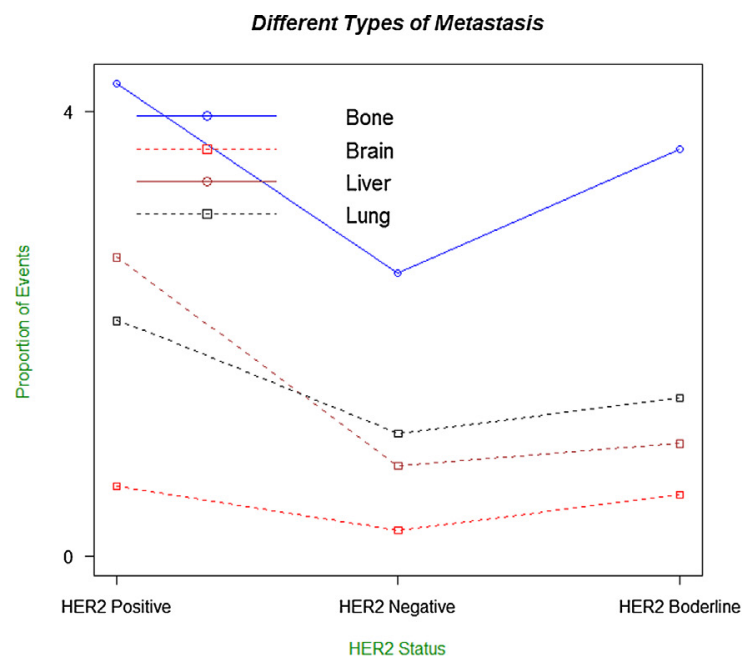

A

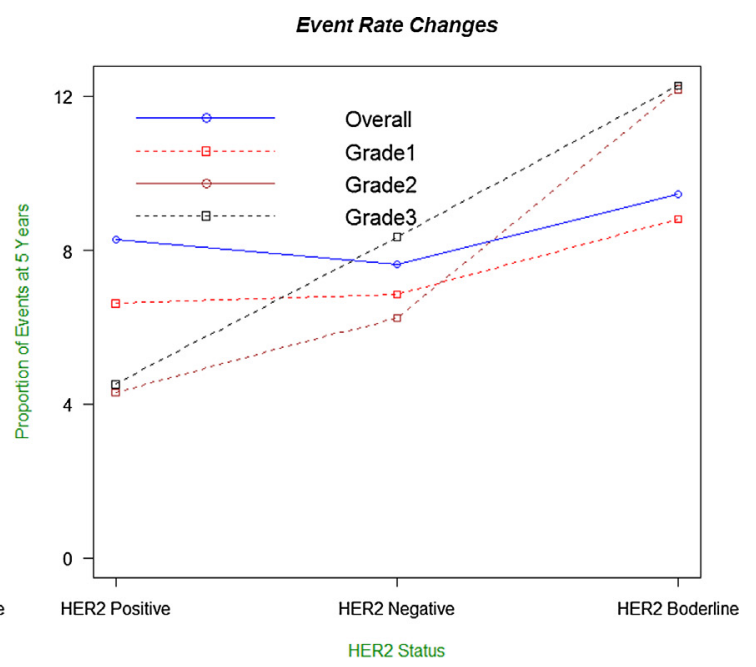

B

Fig. 1. Comparison of different types of metastasis and death occurrence in HER2 Positive, HER2Negative, and HER2 Borderline. 

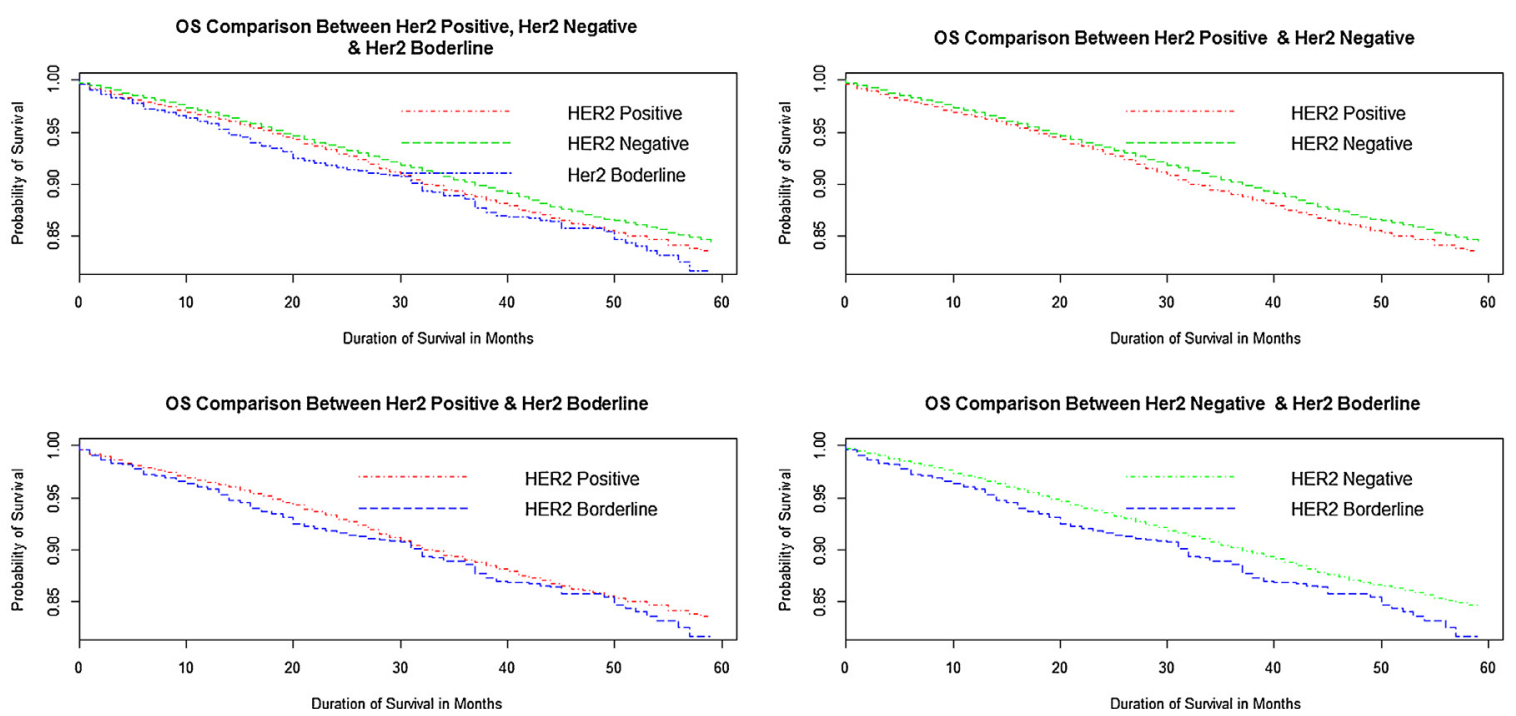

Fig 2. Comparison of Overall Survival(OS) betweenHER2 Positive, HER2Negative and HER2 Borderline.

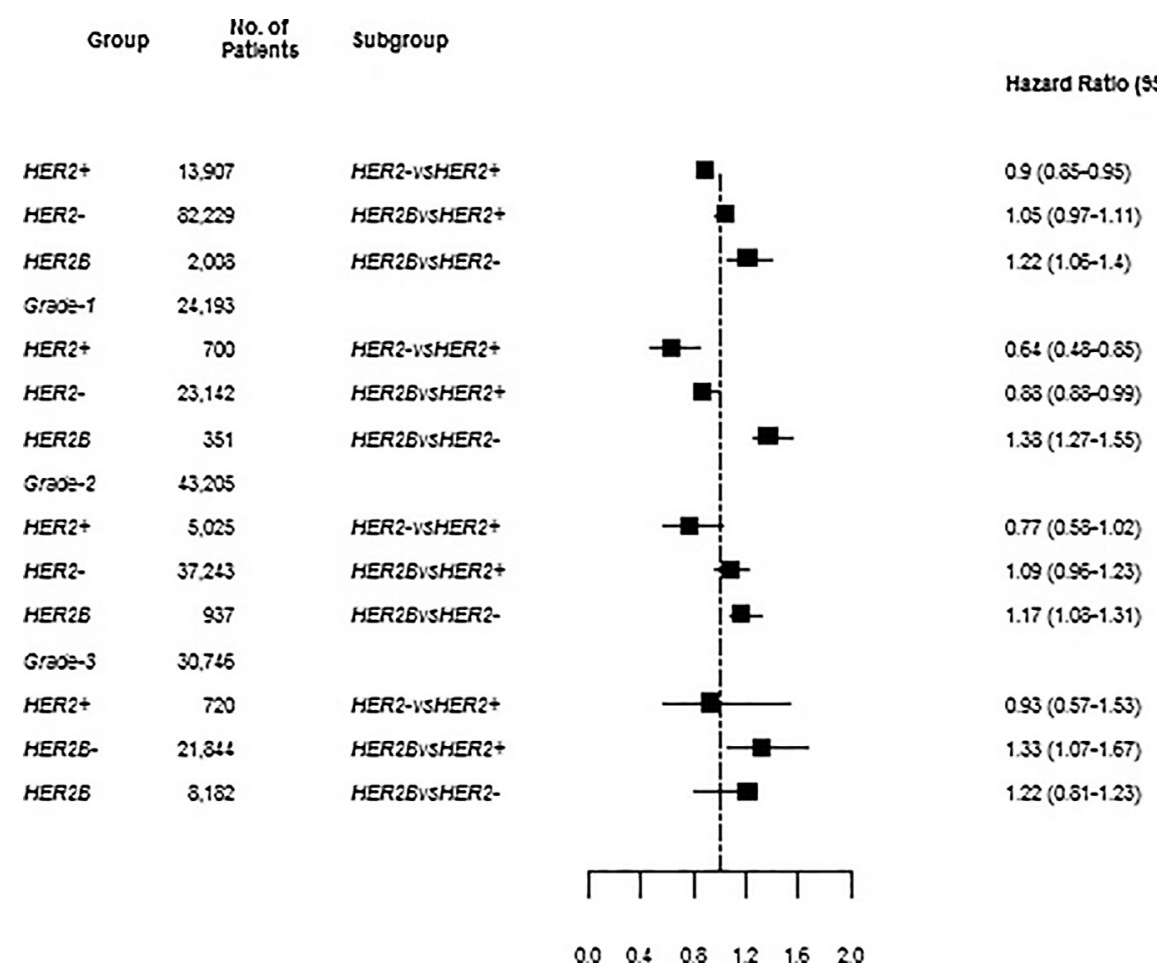

Fig. 3. Forest Plot with Cox PH Model Performance in HER2 Positive, HER2Negative, and HER2 Borderline.

due to the adoption of proportional hazard assumption without their presence. Therefore AFT model with different distributional assumption can be better picked in such circumstance. The AFT models consider a statistical distribution of the duration of survival and avoid the proportional hazards assumption $(\mathrm{PH}) .^{10}$

The Cox proportional hazard model is widely adopted in cancer research, but inference obtained through AFT model is more valid and generates minor bias towards statistical inference. The $\mathrm{PH}$ assumption test can also be avoided in the AFT. The AFT is also reliable where the Cox proportional hazard model assumption is not accepted. In the SEER database patients cause of death information are included as death due cancer and death due other causes. The other causes of death can be defined as competing risk.
It is a situation where more than one cause of death is possible. However, it computationally difficult to get statistical convergence in presence of competing risks. ${ }^{11}$ In this analysis only censored cases and patients died due to cancer are included to carry the competing risk free survival analysis.

The study to explore the complete gene-expression reveals that basal-like, normal-like, luminal-like, and HER-2 positive are four molecular classes for breast cancer. ${ }^{12}$ There is a significant difference of gene-expression is observed between ER-positive and ER-negative patients. ${ }^{13-15}$ The molecular resistance also changes for different chemotherapy. It is also observed that HER 2 positive is more sensitive to chemotherapy. ${ }^{12,16}$ It is important to establish that many of these correlations are present among 
Table 3

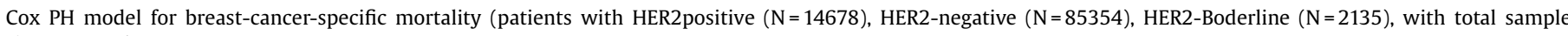
size $=102167$ ).

\begin{tabular}{|c|c|c|c|c|c|}
\hline Effect & Model & HR/Coefficient (95\% CI) & P-value & Number of Event at the end of 5 years & $\%$ of Event \\
\hline \multirow[t]{3}{*}{ HER2-vsHER2+ } & Model1 & $0.90(0.85,0.95)$ & 0.001 & 48 & 6.49(HER2+) \\
\hline & Model2 & $0.08(0.02,0.14)$ & 0.006 & & \\
\hline & Model3 & $0.07(0.02,0.13)$ & 0.006 & & \\
\hline \multirow{3}{*}{ HER2BvsHER2+ } & Model1 & $1.05(0.97,1.13)$ & 0.176 & 1012 & 14.24(HER2-) \\
\hline & Model2 & $0.05(-0.12,0.02)$ & 0.158 & & \\
\hline & Model3 & $-0.04(-0.11,0.02)$ & 0.175 & & \\
\hline \multirow[t]{3}{*}{ HER2BvsHER2- } & Model1 & $1.22(1.06,1.40)$ & 0.004 & 16 & 15.06(HER2B) \\
\hline & Model2 & $-0.19(-0.33,-0.05)$ & 0.007 & & \\
\hline & Model3 & $-0.16(-0.29,-0.04)$ & 0.008 & & \\
\hline \multicolumn{6}{|l|}{ Grade1 } \\
\hline \multirow[t]{3}{*}{ HER2- vsHER2+ } & Model1 & $0.64(0.48,0.85)$ & 0.002 & 351 & 1.20(HER2+) \\
\hline & Model2 & $0.24(-0.03,0.53)$ & 0.085 & & \\
\hline & Model3 & $0.3751(0.1419,0.6082)$ & 0.001 & & \\
\hline \multirow[t]{3}{*}{ HER2BvsHER2+ } & Model1 & $0.88(0.79,0.99)$ & 0.035 & 2381 & 0.14(HER2-) \\
\hline & Model2 & $0.47(0.18,0.76)$ & 0.008 & & \\
\hline & Model3 & $0.2165(-0.0282,0.4612)$ & 0.082 & & \\
\hline \multirow[t]{3}{*}{ HER2B vsHER2- } & Model1 & $1.38(1.27,1.5)$ & 0.000 & 80 & 1.36(HER2B) \\
\hline & Model2 & $0.02(-0.46,0.51)$ & 0.923 & & \\
\hline & Model3 & $0.03(-0.35,0.42)$ & 0.859 & & \\
\hline \multicolumn{6}{|l|}{ Grade2 } \\
\hline \multirow[t]{3}{*}{ HER2- vsHER2+ } & Model1 & $0.77(0.58,1.02)$ & 0.076 & 733 & 2.78(HER2 +) \\
\hline & Model2 & $-0.09(-0.22,0.02)$ & 0.123 & & \\
\hline & Model3 & $0.08(-0.01,0.18)$ & 0.095 & & \\
\hline \multirow[t]{3}{*}{ HER2BvsHER2+ } & Model1 & $1.09(0.96,1.23)$ & 0.164 & 2723 & 2.22(HER2-) \\
\hline & Model2 & $0.09(-0.01,0.21)$ & 0.104 & & \\
\hline & Model3 & $-0.08(-0.19,0.02)$ & 0.134 & & \\
\hline \multirow[t]{3}{*}{ HER2B vsHER2- } & Model1 & $1.17(1.08,1.31)$ & 0.003 & 91 & 5.47(HER2B) \\
\hline & Model2 & $-0.29(-0.51,-0.06)$ & 0.012 & & \\
\hline & Model3 & $-0.24(-0.43,-0.04)$ & 0.014 & & \\
\hline \multicolumn{6}{|l|}{ Grade3 } \\
\hline \multirow[t]{3}{*}{ HER2- vsHER2+ } & Model1 & $0.93(0.57,1.53)$ & 0.792 & 1237 & 1.95(HER2 + ) \\
\hline & Model2 & $-0.16(-0.27,-0.05)$ & 0.004 & & \\
\hline & Model3 & $-0.33(-0.42,-0.25)$ & 0.001 & & \\
\hline \multirow[t]{3}{*}{ HER2BvsHER2+ } & Model1 & $1.33(1.07,1.67)$ & 0.010 & 663 & 10.83(HER2-) \\
\hline & Model2 & $-0.30(-0.37,-0.22)$ & $<.001$ & & \\
\hline & Model3 & $-0.14(-0.25,-0.04)$ & 0.004 & & \\
\hline \multirow[t]{3}{*}{ HER2B vsHER2- } & Model1 & $1.22(0.81,1.23)$ & 0.998 & 207 & 5.44(HER2B) \\
\hline & Model2 & $0.01(-0.19,0.22)$ & 0.891 & & \\
\hline & Model3 & $0.01(-0.17,0.20)$ & 0.861 & & \\
\hline
\end{tabular}

${ }^{*}$ HER2+ =HER2 Positive, ${ }^{* *}$ HER2 $-=$ HER2 Negative, ${ }^{* * *}$ HER2Boderline = HER2B.

molecular class and conventional histo-pathological variables. This study reveals that HER 2 borderline is clinically relevant. There is a further in-depth study on HER 2 borderline is required to explore.

\section{Conclusion}

Data Explorations are a foundation of our ability to identify any factor towards contribution against cancer. Data exploration through consideration/adjustment of different subtypes is important. In addition, it is required to take new initiative towards research and prevention to make control over death due to specific reason in cancer like HER2 borderline. This SEER program data can be explored in future as well to detect the change on OS due to HER2 status among the patients.

\section{Conflict of interest}

None.

\section{Acknowledgements}

The authors thank the two anonymous referees for their cautious reading and constructive suggestions which have led to improvement on earlier versions of the manuscript.

\section{Appendix A. Supplementary data}

Supplementary data associated with this article can be found, in the online version, at https://doi.org/10.1016/j.cegh.2017.12.005.

\section{References}

1. Zhu W, Perez EA, Hong R, et al. Age-related disparity in immediate prognosis of patients with triple-negative breast cancer: a population-based study from seer cancer registries. PLoS One. 2015;10:e0128345.

2. DeSantis CE, Siegel RL, Sauer AG, Miller KD, Fedewa SA, Alcaraz KI, et al. Cancer statistics for African Americans, 2016: Progress and opportunities in reducing racial disparities. CA Cancer J Clin. 2016;66:290-308.

3. Aaronson NK, Ahmedzai S, Bergman B, et al. The European Organization for Research and Treatment of Cancer QLQ-C30: a quality-of-life instrument fo use in international clinical trials in oncology. J Natl Cancer Inst. 1993;85:365376.

4. Meunier F, General D. European Organisation for Research and Treatment of Cancer (EORTC): the challenges of academic cancer clinical research in Europe. Eur Oncol Haematol. 2005;2005.

5. Malvezzi M, Bertuccio P, Levi F, et al. European cancer mortality predictions for the year 2013. Ann Oncol. 2013;24:792-800.

6. Pauletti G, Dandekar S, Rong H, et al. Assessment of methods for tissue-based detection of the HER-2/neu alteration in human breast cancer: a direct comparison of fluorescence in situ hybridization and immunohistochemistry. $J$ Clin Oncol. 2000;18:3651-3664.

7. Xia D, Biology L of C, National Cancer Institute, Center for Cancer Researc, National Institutes of Health, Bethesda, et al. Recent Advances in Understanding the Structure and Function Relationship of Multidrug 
Resistance-Linked ABC Transporter P-glycoprotein. J Can Res Updates. 2016;5:88-98.

8. Bhattacharyya T, Bhattacharjee A, Zhu W, et al. Competing risk: an illustration with aspiration pneumonia in head and neck cancer patients undergoing radical radiotherapy: a biostatistician's perspective. Indian J Cancer. 2014;51 (4):406-409.

9. Altman DG, De Stavola BL, Love SB, et al. Review of survival analyses published in cancer journals. Br J Cancer. 1995;72:511-518.

10. Prabhash K, Patil V, Noronha V, et al. Bayesian accelerated failure time and its application. Chemother Drug Treat Trial. 2016;17(4):671-690.

11. Bhattacharjee A. Bayesian competing risks model: an application to breast cancer clinical trial with incomplete observations. J Stat Manage Syst. 2015;18 (4):381-404.

12. Sørlie T, Perou CM, Tibshirani R, et al. Gene expression patterns of breast carcinomas distinguish tumor subclasses with clinical implications. Proc Natl Acad Sci U S A. 2001;98:10869-10874.
13. Pusztai L, Ayers M, Stec J, et al. Gene expression profiles obtained from fineneedle aspirations of breast cancer reliably identify routine prognostic markers and reveal large-scale molecular differences between estrogennegative and estrogen-positive tumors. Clin Cancer Res AACR. 2003;9:24062415.

14. Sotiriou C, Neo S-Y, McShane LM, et al. Breast cancer classification and prognosis based on gene expression profiles from a population-based study. Proc Natl Acad Sci U S A. 2003;100:10393-10398.

15. Sørlie T, Tibshirani R, Parker J, et al. Repeated observation of breast tumor subtypes in independent gene expression data sets. Proc Natl Acad Sci U S A. 2003;100:8418-8423.

16. Rouzier R, Perou CM, Symmans WF, Ibrahim N, et al. Breast cancer molecular subtypes respond differently to preoperative chemotherapy. Clin Cancer Res. 2005;11:5678-5685. 